\title{
Nanotechnology: Conservative Dentistry and Endodontics
}

\author{
Dr. Dayanand Chole ${ }^{1}$, Dr. Irshad Khan ${ }^{2}$, Dr. Shashank Kundoor ${ }^{3}$, \\ Dr. Srinivas Bakle ${ }^{4}$, Dr. Neha Gandhi ${ }^{4}$, Dr. Rucha Deshpande ${ }^{5}$ \\ ${ }^{I}$ Professor and Head: Department of Conservative Dentistry and Endodontics \\ P.D.U Dental College, Solapur \\ ${ }^{2}$ Post Graduate Student: Department Of Conservative Dentistry and Endodontics \\ PDU Dental College, Solapur \\ ${ }^{3 / 4}$ Reader: Department Of Conservative Dentistry and Endodontics \\ PDU Dental College, Solapur \\ ${ }^{5,6}$ Senior Lecturer: Department Of Conservative Dentistry and Endodontics \\ PDU Dental College, Solapur
}

\begin{abstract}
Nanotechnology deals with the physical, chemical, and biological properties of structures and their components at nanoscale dimensions. Nanotechnology is based on the concept of creating functional structures by controlling atoms and molecules on a one-by-one basis. The use of this technology will allow many developments in the health sciences as well as in materials science, bio-technology, electronic and computer technology, aviation, and space exploration. The science of nanotechnology has become the most popular area of research, currently covering a broad range of applications in dentistry. This review describes the basic concept of nanomaterials, recent innovations in nanomaterials and their applications in restorative dentistry. Advances in nanotechnologies are paving the future of dentistry, and there are a plenty of hopes placed on nanomaterials in terms of improving the health care of dental patients.
\end{abstract}

Keywords: Nanotechnology, Nanodentistry, Nanomaterials, Nanocomposite

\section{Introduction}

Nanotechnology era is fast approaching which was unheard two decades ago. The growing interest in this field is giving emergence to new field called Nanodentistry, a science \& technology of diagnosing, treating \& preventing diseases, and preserving \& improving human health, using nanoscale structured materials. The mechanism on which nanodentistry works is by constructing microsized dental nanorobots which use specific motility mechanisms to crawl or swim through human tissue with navigational precision, acquire energy, sense and manipulate their surroundings, achieve safe cytopenetration and use any of the multitude techniques to monitor, interrupt, or alter nerve impulse traffic in individual nerve cells in real time. These nanorobots function may be controlled by an on board nanocomputer that executes pre programmed instructions in response to local sensor stimuli.The concept of nanotechnology introduced in 1959 by late Nobel Physicist Richard P Feynman in dinner talk said, "There is plenty of room at the bottom," proposed employing machine tools to make smaller machine tools, these are to be used in turn to make still smaller machine tools, and so on all the way down to the atomic level, noting that this is "a development which I think cannot be avoided". ${ }^{2}$ The word "nano," which is derived from the Greek word (nannos) meaning "dwarf," is a prefix that literally refers to 1 billionth of a physical size. ${ }^{3}$ According to the definition of the National Nanotechnology Initiative, nanotechnology is the direct manipulation of materials at the nanoscale. ${ }^{4}$

Nanotechnology will give us the ability to arrange atoms as we desire and subsequently to achieve effective, complete control of the structure of matter. ${ }^{5}$ Nanotechnology is based on the idea of creating functional structures by controlling atoms and molecules on a one-by-one basis. What makes nanoparticles interesting and bestows unique features upon them is the fact that their size is smaller than the critical lengths defining many physical events. In general, nanotechnology is translated as "the science of the small". 6

\section{Approaches in nanotechnology:}

- Bottom-up approach: Seeks to arrange smaller components into more complex assemblies, the covalent bonds of which are extremely strong. ${ }^{8}$

- Top- Down approach: Seeks to produce smaller devices by using larger ones in achieving precision in structure and assembly. ${ }^{8}$

- Functional approach: Seeks to develop components of a desired functionality without regard to how they might be assembled. ${ }^{9}$ 
- Biomimetic Approach: Seeks to apply biomolecules for applications in nanotechnology. ${ }^{(9)}$

- Nanocomposite:

\section{Nanotechnology in conservative dentistry:}

Microfillers in composites and microcore materials have long been in use. Although the filler particle size cannot be reduced below $100 \mathrm{~nm}$, nanocomposite particles are minute enough to be synthesized at the molecular level. These nanoparticles improve the compressive strength of the material. Filler particles of submicron size, such as zirconium dioxide, are also necessary to improve polishability and esthetics. ${ }^{10}$

Non-agglomerated discrete nanoparticles that are homogeneously distributed in resins or coatings to produce nanocomposites have been successfully manufactured by Nanoproducts Corporation. The nanofiller used: Aluminosilicate powder with a mean particle size of 80 ran 1:4 M ratio of alumina to silica and a refractive index of 1.508 . These nanocomposites have superior hardness, flexural strength, modulus of elasticity, decreased polymerization shrinkage and also have excellent handling properties. ${ }^{11} \mathrm{~A}$ recent study by Xu et al. has evaluated the incorporation of nanosized $\mathrm{CaPO}_{4}$ particles into resin based-composites, with a resulting improvement in stress bearing capacity as well as ion release that could inhibit dental caries. ${ }^{12}$

\section{- Coating agents:}

These agents contain light activated nanosized fillers which can be used as coating over the composite, glass inomer cements, jacket crowns and veneers. Incorporation of nanofillres provide superb polish on the restorations which prevents staining, increases abrasion and wear resistance. ${ }^{30}$

\section{- Nanobond:}

It is a nano particulate reinforced adhesive system. These new bonding agents are prepared from nano solutions which contain homogenous nanoparticles dispersed in the solution. Silica nanofillers are stable and do not cluster in the solution so provide the superior bond strength values. Nano interaction zone (NIZ - <300 nm) with minimal decalcification and almost no exposure to collagen fibers producing an insoluble calcium compound for a better bond less likely to deteriorate from enzymes contained in the mouth. ${ }^{14}$

\section{- Nanosolution:}

Nanosolutions produce unique and dispersible nanoparticles, which can be used in bonding agents. This ensures homogeneity and ensures that the adhesive is perfectly mixed everytime. ${ }^{15}$

\section{- Dental hypersensitivity}

Reconstructive dental nanorobots, using native biological materials, could selectively and precisely occlude specific tubules within minutes, offering patients a quick and permanent cure. ${ }^{16}$ On reaching the dentin, the nanorobots enter dentinal tubular holes that are 1 to $4 \mu \mathrm{m}$ in diameter and proceed toward the pulp, guided by a combination of chemical gradients, temperature differentials and even position of navigation, all under the control of the onboard nanocomputer as directed by the dentist. ${ }^{17,18}$

\section{- Nanoanesthesia:}

Nanorobots will play a powerful role in the induction of local anesthesia in the era of robots will be instilled on the patient's gingival that will result in anesthesia. Anesthesia is fast acting, and reversible, with no side effects or complications with its use. ${ }^{19}$

\section{- Dental Durability and Cosmetics:}

Covalently bonded artificial materials such as, sapphire or diamond in a fracture resistant nanostructured composite material that possibly include carbon nanotubes are used for replacing upper enamel layers for aesthetic purposes. ${ }^{20}$

\section{- Nano Impression Materials:}

Nanofillers are integrated in vinylpolysiloxanes, producing a unique edition of siloxane impression material. The material has a better flow, improved hydrophilic properties, tear strength and enhanced detail precision. The presence of the nanostructure increases the fluidity of the material, especially when pressure is applied. ${ }^{19,21}$

\section{- Nano toothpaste:}

Nano-Whitening Toothpaste is toothpaste that contains synthesized hydroxyapatite, a key component of tooth enamel, as nanosized crystals. It has been proven to freshen breathe as well as whiten teeth. This toothpaste contains ingredents such as: 
Patented nano technology aka Nanoxyd, Calcium peroxide,Contains Enzymes such as (papain and bromelain), Fluoride combination, Co-enzyme Q10 and Vitamin E. ${ }^{22}$

\section{- Nanorobotic Dentifrice (Dentifrobots):}

Sub occlusal dwelling nano robotic dentifrice delivered by mouthwash or toothpaste could patrol all supra gingival and sub gingival surfaces at least once a day, metabolising trapped organic matter into harmless and odourless vapours and performing continuous calculus debridement. These invisibly small dentifrobots [1-10 micon], crawling at 1-10 microns/sec, would be inexpensive, purely mechanical devices, that would safely deactivate themselves if swallowed and would be programmed for better cleaning of the teeth. ${ }^{23}$

\section{- Cavity Preparation and Restoration:}

Multiple nano robots working on the teeth in unison, invisible to the naked eye, may be used for cavity preparation and restoration of teeth. The cavity preparation is very precisely restricted to the demineralised enamel and dentin, thus providing maximum conservation of sound tooth structure. Further the devices would identify particles of food, plaque or tartar and lift them from the teeth to be rinsed away. Being suspended in liquid and able to swim about, devices would be able to reach surfaces beyond reach of toothbrush bristles or the fibres of floss. Sub-occlusally dwelling nano robots delivered by dentifrice patrol all supra-gingival and subgingival surfaces metabolizing trapped organic matter performing continuous calculus debridement. They prevent tooth decay and provide a continuous barrier to halitosis with good deformability, corrosion resistance, and surface finish. ${ }^{23}$

\section{- Nanofilled glass ionomer cement:}

Nano glass ionomers are designed to meet the various requirements, same as other materials used in the mouth. Nanotechnology was used in the development to provide some value added features not typically associated with glass ionomer restorative materials. By using bonded nanofillers and nanocluster fillers, along with FAS glass newer type of GIC was formulated using nanotechnology along with its fluoride releasing property. This product meets a wide range of clinical indications ranging from Class I, II, V and core buildup. Nano GIC is an ideal restorative material for everyday dentistry. Advantages of this material are: superb polish, excellent esthetics, higher wear resistance, It is faster, easier to mix and dispense. ${ }^{24,25}$

\section{- Nanotech floss:}

Ultra-thin, ultra-glide, completely non-shredding with excellent tensile strength. The unique nanostructure of dental tape allows for the addition of flavours, and delivery of medications. ${ }^{26}$

\section{- Esthetics by ultrafine polishing:}

Polishing the teeth results in roughness, this provides medium for biofilm formation. Ultra-fine polishing of teeth leads to nanoscale roughness which is few in nanometers. It protects the teeth from cariogenic bacteria, which can be easily removed from these ultrafine polished surfaces, therefore prevent staining and lead to superior esthetics of the restorations. ${ }^{24,14}$

\section{- Nano-ceramics:}

The Organically Modified Ceramic nano-particles comprise a polysiloxane backbone. These NanoCeramic particles can be best described as inorganic-organic hybrid particles where the inorganic part consisting of siloxane and the methacrylic organic part blends all the particles with resin matrix. The good resistance to microcrack propagation might be related to the strengthening effect of the nano-ceramic particles. Propagating cracks are either more often reflected or absorbed by the nanoceramic particles. ${ }^{27,28}$

\section{- Nanotechnology microscope:}

The new developed deep probe detectors consisting of the electromagnetic spectrum will be available to screen the human body to reveal hidden matter such as, deep tumors and occult caries in teeth. This is known as Terahertz radiation, which lies in between light and radio waves in the spectrum. ${ }^{29}$

\section{- Nanozone:}

Nano technology based ozone therapy. It provides strongly oxidizing ozone. When given in adequate doses allows removal of $99.9 \%$ of bacteria which are responsible for the development of dental caries. $^{30}$ 


\section{- Major tooth repair:}

Tooth structure loss is treated by various filling materials. These days we use nanofilled composites resembling as close as tooth structure. LET'S DREAM MORE... How about replacing the damaged tooth with natural tooth developed in vitro and placed in tooth socket using nanorobots. This may be the dream of today but may be reality of tomorrow. Both mineral and cellular components would be regenerated and this will be made possible by nanodentistry along with tissue engineering. ${ }^{31}$

\section{- Nano reminerializing agent:}

Calcium Nanophosphate paste (Desensibilize Nano-P, FGM Produtos Odontologicos) for bleached enamel. The calcium nanophosphate crystals may have penetrated more deeply into the defects of the enamel, forming a "reservoir-like" deposit of the eroded calcium and phosphate ions. The reservoir like deposit help maintain a state of supersaturation with enamel minerals. ${ }^{32}$

\section{- Nanoparticles of gold and silver:}

Silver and gold nanoparticles are being used as an alternative to dental filling agents. The unique advantages of these nanoparticles are the anti wear; antibacterial and antifungal properties enable their use in restorative dentistry. They also act on dental biofilms in root canals when used during endodontic therapy. ${ }^{33}$

\section{Nanotechnology in endodontics: \\ - Bone replacement Materials:}

Chen et al. took advantage of these latest developments in the area of nanotechnology to simulate the natural biomineralization process to create the hardest tissue in the human body, dental enamel, by using highly organized microarchitectural units of nanorod-like calcium hydroxyapatite crystals arranged roughly parallel to each other.Hydroxyapatite nanoparticles used to treat bone defects are;

Ostim ${ }^{\circledR}$ (osartis GmbH Germany) HA. •

VITOSS $®$ (orthovita Inc, USA) HA+ TCP. •

NanOssTM (Angstrom Medica ,USA) HA. ${ }^{34}$

\section{- Nanoneedles \& tweezers:}

Nanoneedles may be conical or tubular needles in the nanometer size range, made from silicon or boron-nitride with a central bore of sufficient size to allow the passage of large molecules. The conical needles can be mounted in arrays and used to deliver any drug or vaccination antigen. In 2005 the Research Institute for Cell Engineering at Japan's National Institute of Advanced Industrial Science and Technology(AIST) and Tokyo University of Agriculture and Technology used nanoneedles controlled by an atomic force microscope(AFM) to penetrate the nucleus of living cells and insert molecules of nucleic acid, proteins or possibly to carry out cell surgery. These will make cell surgery possible in the future. ${ }^{35}$

\section{- Endodontic Sealer:}

The applications of nanotechnology are not limited to filling materials but have been extended to endodontic applications. A bioceramic based nanomaterials (EndoSequence BC sealer) composed of calcium silicate, calcium phosphate, calcium hydroxide, zirconia and a thickening agent, has been developed recently. Nanoparticles have improved the handling and physical properties. During the hydration reaction in the root canal, a nanocomposites structure of calcium silicate and hydroxyapatite is formed. The hydration reaction and setting time is affected by the availability of water and setting time may be prolonged in overly dried canals. Nano sized particles facilitate delivery of material from 0.012 capillary needle and adopt to irregular dentin surfaces. It sets hard in a matter of a few hours providing excellent seal and dimensional stability. Upon setting, it forms of hydroxyapatite; providing biocompatible and bioactivity. The highly alkaline $\mathrm{pH}$ (12.8) gives antimicrobial properties as well .Another example is a silicon based sealer (Gutta-Flow Sealer) with an addition of gutta-percha powder and silver nanoparticles. This material is available in the form of uni-dose capsule that can be mixed and injected. This nano-sealer has good biocompatibility, dimensionally stable and sets within half an hour. This material has been reported to improve the sealing capability and better resistance to bacterial penetration. For infection point of view, the antibacterial activity of endodontic sealers can be very beneficial. Recently, antibacterial quaternary ammonium polyethyleneimine (QPEI) nanoparticles have been incorporated into existing sealers such as $\mathrm{AH}$ plus, Epiphany and Guttaflow . Resin composites containing QPEI nanoparticles resulted in prolonged antibacterial activity without compromising the mechanical properties. In order to obtain similar antibacterial effect in endodontic sealers, $0-2$ wt $\%$ QPEI nanoparticles were added in to the commercially available sealers. The addition of QPEI 
nanoparticles is very stable, leaching no byproducts in the surrounding and there was no effect on the biocompatibility; however, the antibacterial properties remained excellent. ${ }^{13}$

\section{- Denbur Nano-Brush:}

It is an innovative applicator that follows the natural anatomy to access the root canal. It can be used to remove the pulp from the chamber to clean the debris in the canal, or to apply cleansing and restorative materials onto the internal radicular surfaces in the canal. It promotes deep penetration of sealers into the dentinal tubules and lateral canals. ${ }^{36}$

\section{- Nanoparticles as antimicrobial agents:}

Nano particulates exhibit higher antibacterial activity as a result of their poly cationic or poly anionic in nature with higher surface area and charge density, resulting in a greater degree of interaction with the bacterial cell. These disinfect the canal by removing the residual microbes in the canal and enhance the antibacterial action of the intra canal medicaments. Nano particles have been applied in the treatment of bacterial bio films as well as wound healing primarily because of their antimicrobial properties and biocompatibility. These have been shown to provide a significant improvement in the root canal disinfection by effectively eliminating the residual adherent and non adherent bacteria as well as increasing the diffusion of antibacterial components from the root canal sealers. There is significant antibacterial efficacy against Enterococcus faecalis. Nano care plus is used for final rinsing of root canal treatment. It has Bacteriostatic effect. ${ }^{37}$

\section{Conclusion}

Nanodentistry will give a new vision to comprehensive oral health care, as now trends of oral health have been changing to more preventive intervention than a curative and restorative procedure. This science might sound like a fiction now, but Nanodentistry has a strong potential to revolutionize dentistry as to diagnosing and treating dental diseases in future. It opens up new avenues for vast, abundant research. Nanotechnology will change dentistry, health care and human life more profoundly than other developments.

\section{References}

[1]. Chandra Mouli PE , Manoj Kumar S , P arthiban S ,International Journal of Biological \& Medical Research , Nanotechnology in Dentistry - A Review . 2012; 3(2): 1550-1553

[2]. Feynman RP.There is plenty of room at the bottom Eng Sci 1960;23:22-36

[3]. Saravana KR, Vijayalakshmi R. Nanotechnology in dentistry. Ind J Dent Res. 2006;17:62-65

[4]. Kong LX, Peng Z, Li SD, Bartold M. Nanotechnology and its role in the management of periodontal diseases. Periodontol 2000. 2006;40:184-196.

[5]. Rieth M. Nano-Engineering - Studies and Conclusions Nano-Engineering in science and technology. 1st ed. Singapore: World Scientific Publishing Co. Ptc. Ltd; 2003. pp. 91-103

[6]. Duke ES. Has dentistry moved into the nanotechnology era? Compend Contin Educ Dent. 2003;24:380-382

[7]. Mallanagouda P, Dhoom S. M, Sowjanya G. Future impact of nanotechnology on medicine and dentistry. J. Ind. Soc. Periodontol. 2008;12:34-40

[8]. Das S, Gates AJ, Abdu HA, Rose GS, Picconatto CA and Ellenbogen JC., 2007. "Designs for Ultra-Tiny,Special-Purpose Nanoelectronic Circuits". IEEE Transactions on Circuits and Systems I (11):2528-2540.

[9]. SNEHA SUNDAR RAJAN , SHASHI RASHMI ACHARYA AND VIDYA SARASWATHI . NANODENTISTRY Indian J.Sci.Res. 4(2) : 233-238, 2013.

[10]. Dr. Mehra , Dr. Nabhi K. A Nanorobotics - The Changing Face of Dentistry . IJSR Volume 5 Issue 3, March 2016.

[11]. Sivaramakrishnan SM, Neelakantan P (2014) Nanotechnology in Dentistry - What does the Future Hold in Store? Dentistry 4: 198.

[12]. Xu HH, Sun L, Weir MD, Takagi S, Chow LC, et al. (2007) Effects of incorporating nanosized calcium phosphate particles on properties of whisker-reinforced dental composites. J Biomed Mater Res B Appl Biomater 81: 116-125.

[13]. Zohaib Khurshid, Muhammad Zafar, Saad Qasim. Advances in Nanotechnology for Restorative Dentistry , materials 2015, 8, 717731.

[14]. Khosla R. Nanotechnology in dentistry. Famdent Pract Dent Handb 2009; 9:69-84.

[15]. Rita Chandki , M. Kala , Kiran Kumar N. , Biji Brigit , Priyank Banthia , Ruchi Banthia. 'NANODENTISTRY': Exploring the beauty of miniature. J Clin Exp Dent. 2012;4(2):e119-24.

[16]. Bhavna Jha Kukreja, Vidya Dodwad, Tulika Singh. ROBOTIC DENTISTRY-THE FUTURE IS AT THE HORIZON, Journal of Pharmaceutical and Biomedical Sciences (JPBMS), Vol. 16, Issue 16.

[17]. Freitas Jr RA. Nanodentistry. J Am Dent Assoc 2000; 131:1559-66.

[18]. Freitas R.A. Jr. Exploratory design in medical nanotechnology: A mechanical artificial red cell. Artificial Cells Blood Substitute Immobile Biotechnology 1998;26;30-32.

[19]. Kaur Jasdeep, Kaur Kirandeep, Aggarwal Geeta, Kumar Hari S.L. NANOTECHNOLOGY IN DENTISTRY, World Journal of Pharmacy and Pharmaceutical Sciences Vol 5, Issue 4, 2016.

[20]. Freitas RA., Jr Nanodentistry. J Am Dent Assoc, 2000; 131: 1559-65.

[21]. Kumar Saravana R and Vijayalakshmi R, Nanotechnology in Dentistry. Ind J Dent Res, 2006; $17(2): 62$-65.

[22]. Sumit Mohan, Anuraag Gurtu,Anurag Singhal, Ankita Mehrotra. Nanotechnology : Its Implications in Conservative Dentistry and Endodontics, Journal of Dental Sciences \& Oral Rehabilitation 2013; July - September.

[23]. Biji Balan, Sreejesh Narayanan. NANO ROBOTICS - ITS TIME FOR CHANGE, IJOCR Jul - Sep 2014 ; Volume 2 Issue 5. 
[24]. Sasalawad, et al. Nanodentistry: The next big thing is small, International Journal of Contemporary Dental and Medical Reviews(2014).

[25]. Bayne SC. Dental biomaterials: where are we and where are we going? J Dent Educ 2005;69:571-85.

[26]. Saravana KR, Vijayalakshmi R. Nanotechnology in dentistry. Indian J Dent Res 2006;17:62-5.

[27]. Ceram x nanoceramic restorative: Scientific compendium.

[28]. Atabek D, Sillelioglu H, Olmez A. Th e effi ciency of a new polishing material: nanotechnology liquid polish. Oper Dent 2010;35:362-9.

[29]. Rajan MS. Nano: The Next Revolution. $1^{\text {st }}$ ed. New Delhi: National Book Trust; 2011.

[30]. Nanotech Endo and Nanozon at IDS 2013 in Cologne.

[31]. Munish Goel., et al."Nanodentistry - "Unbounding The Future”. EC Dental Science 4.1 (2016): 727-732.

[32]. Fabiola Galbiatti, Veruska Lima.protective effect of calcium nanophosphate and CPP-ACP agents on enamel erosion. Braz.oral.res.2013; 27:463-470.

[33]. Garcia C. R, Augeta F. C, Mejija R. C, Jimenez M. R, Cuevas G. S, et al. Perspectives for the use of silver nanoparticles in dental practice. Int Dent J 2011; 61:297-301.

[34]. Chen HF, Clarkson BH, Sunk, Mansfield JF. Self assembly of synthetic hydroxyapatite nanorods into enamel prism like structure. J Colloid Interface Sci 2005;288(1);97-103.

[35]. Kumar PS, Kumar S, Savadi RC, John J. Nanodentistry: A paradigm shift- from fiction to reality . J Indian Prosthodont Soc 2011:11(1):1-6.

[36]. Waltimo T, Brunner TJ, Vollenweider M, Stark WJ, Zehnder M. Antimicrobial effect of nanometric bioactive glass 45S5. J Dent Res.2007; 86:754-7.

[37]. Shrestha A, Fong SW, Khoo BC, Kishen A. Delivery of antibacterial nanoparticles into dentinal tubules using high intensity focused ultrasound. J Endod 2009; 35:1028-1033. 